\title{
Finite-state self-similar actions of nilpotent groups
}

\author{
Ievgen V. Bondarenko, Rostyslav V. Kravchenko*
}

November 18, 2018

\begin{abstract}
Let $G$ be a finitely generated torsion-free nilpotent group and $\phi: H \rightarrow G$ be a surjective homomorphism from a subgroup $H<G$ of finite index with trivial $\phi$-core. For every choice of coset representatives of $H$ in $G$ there is a faithful self-similar action of the group $G$ associated with $(G, \phi)$. We are interested in what cases all these actions are finite-state and in what cases there exists a finite-state self-similar action for $(G, \phi)$. These two properties are characterized in terms of the Jordan normal form of the corresponding automorphism $\widehat{\phi}$ of the Lie algebra of the Mal'cev completion of $G$.
\end{abstract}

Mathematics Subject Classification 2000: 20F65, 20F18

Keywords: self-similar action, nilpotent group, finite-state action, automaton group

\section{Introduction}

An action of a group $G$ on the set $X^{*}$ of words over a finite alphabet $X$ is called self-similar if for every $x \in X$ and $g \in G$ there exist $y \in X$ and $h \in G$ such that $g(x v)=y h(v)$ for all words $v \in X^{*}$. Every self-similar action of a group on $X^{*}$ implies the action of the group by automorphisms on the $|X|$-regular rooted tree with vertex set $X^{*}$ and edges $(v, v x)$ for all $v \in X^{*}, x \in X$. In terms of automorphisms of rooted trees, self-similarity of the action means that the action of every element of the group "inside" every $|X|$-regular rooted subtree can be represented by an element of the group. Self-similar group actions appear naturally in many areas of mathematics and have applications to holomorphic dynamics, fractal geometry, combinatorics, automata theory, etc. (see [11] and the references therein).

The theory of self-similar group actions can be regarded as the study of positional numeration systems on groups. Bases in these number systems are virtual endomorphisms of groups. A virtual endomorphism of a group $G$ is a homomorphism $\phi: H \rightarrow G$ from a

*This author was supported by the NSF grant number 0503688 and the ERC starting grant GA 257110 TRaWGY. 
subgroup $H<G$ of finite index to $G$. To produce a self-similar action of the group $G$ with "base" $\phi$ we need to choose a set $D$ of coset representatives of $H$ in $G$ called a digit set. Let us enumerate the elements of $D$ by the letters of an alphabet $X$ (here $|X|=[G: H]$ and $\left.D=\left\{q_{x}, x \in X\right\}\right)$. The self-similar action of $G$ on the space $X^{*}$ associated to the triple $(G, \phi, D)$ is constructed as follows. Every element of the group stabilizes the empty word. For every $x \in X$ and $v \in X^{*}$ the action of an element $g \in G$ is defined recursively by the rule

$$
g(x v)=y h(v) \text { with } h=\phi\left(q_{y}^{-1} g q_{x}\right),
$$

where $y \in X$ is the unique letter such that $q_{y}^{-1} g q_{x} \in H$. The constructed action may be not faithful. The kernel of the action does not depend on the choice of the set $D$ and is equal to the maximal normal $\phi$-invariant subgroup of $G$ called the $\phi$-core ([11, Proposition 2.7.5]).

Conversely, one can associate a virtual endomorphism with every faithful self-similar action $\left(G, X^{*}\right)$ as follows. The element $h$ from the definition of self-similar action is called the state of $g$ at $x$ and is denoted by $\left.g\right|_{x}$ (this element is unique if the action is faithful); iteratively we define the state of $g$ at every word by the rule $\left.g\right|_{x_{1} x_{2} \ldots x_{n}}=\left.\left.\left.g\right|_{x_{1}}\right|_{x_{2}} \ldots\right|_{x_{n}}$. For every letter $x \in X$ the stabilizer $S t_{G}(x)$ has finite index in $G$ and then the map $\phi_{x}: S t_{G}(x) \rightarrow G$ given by $\phi_{x}(g)=\left.g\right|_{x}$ is a virtual endomorphism of $G$. If in addition the action $\left(G, X^{*}\right)$ is self-replicating (recurrent), i.e., $\phi_{x}$ is surjective and $G$ acts transitively on $X$, then $\left(G, X^{*}\right)$ can be given by the triple $\left(G, \phi_{x}, D\right)$ for some choice of the digit set $D$.

As a simple example, consider the group $\mathbb{Z}$ with the homomorphism $\phi: 2 \mathbb{Z} \rightarrow \mathbb{Z}$, $\phi(2 a)=a$, and choose the digit set $D=\{0,1\}$, which is also used as an alphabet with a slight abuse in notations. The associated self-similar action corresponds to the binary number system on $\mathbb{Z}$. We have $\left.a\right|_{x_{1} x_{2} \ldots x_{n}}=b$ and $a\left(x_{1} x_{2} \ldots x_{n}\right)=y_{1} y_{2} \ldots y_{n}$ for $a, b \in \mathbb{Z}$ if and only if

$$
a=\left(y_{1}-x_{1}\right)+2\left(y_{2}-x_{2}\right)+2^{2}\left(y_{3}-x_{3}\right)+\ldots+2^{n-1}\left(y_{n}-x_{n}\right)+2^{n} b .
$$

In particular, if $\left.a\right|_{00 \ldots 0}=0$ then the image $a(00 \ldots 0)$ is the usual binary expansion of $a$.

Self-similar group actions are closely related to groups generated by automata. Groups generated by finite automata correspond to finite-state self-similar actions of finitely generated groups. Recall that a faithful self-similar action $\left(G, X^{*}\right)$ is called finite-state if for every $g \in G$ the set of its states $\left\{\left.g\right|_{v}: v \in X^{*}\right\}$ is finite. Then a finitely generated group has a faithful finite-state self-similar action if and only if it can be generated by a finite automaton. One of the fundamental questions in this theory is what groups possess finitestate self-similar actions, i.e., can be realized by finite automata. This property was proved for free abelian groups $\mathbb{Z}^{n}$ [12], Grigorchuk group [7], $G L_{n}(\mathbb{Z})$ [5], lamplighter group [8], free groups and free products of cyclic groups of order 2 [13], Baumslag-Solitar groups $B(1, m)$ [2], certain nilpotent groups [3], etc.

The finite-state self-similar actions of $\mathbb{Z}^{n}$ can be characterized in term of the associated virtual endomorphism as shown by Nekrashevych and Sidki in [12] (see also [11, Theorem 2.12.1]). A virtual endomorphism of $\mathbb{Z}^{n}$ is uniquely extended to a linear operator of $\mathbb{R}^{n}$. Then a faithful self-replicating self-similar action of $\mathbb{Z}^{n}$ with virtual endomorphism $\phi$ 
is finite-state if and only if the spectral radius of $\phi$ is less than 1 . In particular, there is no dependence on the choice of coset representatives.

In this paper we consider self-similar actions of finitely generated torsion-free nilpotent groups. The main goal is to generalize the above mentioned result of Nekrashevych and Sidki. However, self-similar actions of nilpotent groups have a new level of complexity comparing to the actions of abelian groups. For example, a nilpotent group with fixed virtual endomorphism may have a faithful finite-state self-similar action for one choice of coset representatives and be not finite-state for another choice (see example with Heisenberg group in Section 4). Hence we need to answer two questions: Under what conditions on $\phi$ does there exist a finite-state action for $(G, \phi)$ ? Under what conditions does every action for $(G, \phi)$ finite-state?

Let $G$ be a finitely generated torsion-free nilpotent group with surjective virtual endomorphism $\phi: H \rightarrow G$. Since we are interested in faithful self-similar actions of the group $G$, we assume that $\phi$-core is trivial. One can check the triviality of $\phi$-core by the following statement.

Proposition 1 (Corollary 1 in [3]). Let $G, H, \phi$ be as above. If $\phi$-core is trivial, then $\phi$ is injective. If $\phi$ is injective, then $\phi$-core is trivial if and only if the virtual endomorphism $\left.\phi\right|_{Z(H)}: Z(H) \rightarrow Z(G)$ of the center $Z(G)$ of the group $G$ has trivial core.

The proposition implies that $\phi$ is also injective and hence it is an isomorphism. The same proposition says that if we know that $\phi$ is an isomorphism then $\phi$-core is trivial if and only if $\left.\phi\right|_{Z(H)}$-core is trivial. Since $Z(G)$ is abelian, by [11, Proposition 2.9.2] the $\left.\phi\right|_{Z(H)}$-core is trivial if and only if no eigenvalue of $\left.\phi\right|_{Z(H)}$ is an algebraic integer, which can be effectively checked.

By a theorem of Mal'cev (see [10] and the next section) there exists the unique real nilpotent connected and simply connected Lie group L, Mal'cev completion of $G$, such that the group $G$ is a discrete subgroup of $L$ and the topological space $L / G$ is compact. Since $H$ is a subgroup of finite index, the isomorphism $\phi: H \rightarrow G$ lifts to an automorphism of the Lie group $L$ also denoted by $\phi$. Let $\mathcal{L}$ be the Lie algebra of $L$ and denote by $\widehat{\phi}$ the differential of $\phi$ at the identity, which is an automorphism of $\mathcal{L}$. Then the existence of finite-state self-similar action of the group $G$ can be characterized in terms of the Jordan normal form of $\widehat{\phi}$.

Theorem 2. Let $G$ be a finitely generated torsion-free nilpotent group. Let $\left(G, X^{*}\right)$ be a faithful self-replicating self-similar action with virtual endomorphism $\phi$ (associated to some letter $x \in X)$. If the action $\left(G, X^{*}\right)$ is finite-state then the spectral radius of $\widehat{\phi}$ is not greater than 1 and for every eigenvalue of modulus 1 the associated Jordan cells in the Jordan normal form of $\widehat{\phi}$ have size 1 . Conversely, if the automorphism $\widehat{\phi}$ satisfies the previous condition then there exists a finite-state self-similar action of $G$ with virtual endomorphism $\phi$.

One can restate the theorem as follows. Let $\phi$ be a surjective virtual endomorphism of $G$ with trivial core. There exists a digit set $D$ with $e \in D$ such that the self-similar action 
associated to $(G, \phi, D)$ is finite-state if and only if the Jordan normal form of $\widehat{\phi}$ satisfies the condition in the theorem.

Theorem 3. Let $G$ be a finitely generated torsion-free nilpotent group, and let $\phi$ be a surjective virtual endomorphism of $G$ with trivial core. Every self-similar action of $(G, \phi)$ is finite-state if and only if the spectral radius of $\widehat{\phi}$ is less than 1.

In particular, if the Jordan normal form of $\widehat{\phi}$ satisfies the condition in Theorem 2 and $\widehat{\phi}$ has an eigenvalue of modulus 1 , then the pair $(G, \phi)$ possesses both finite-state and nonfinite-state self-similar actions. This situation cannot happen for abelian group $\mathbb{Z}^{n}$, because if a virtual endomorphism of $\mathbb{Z}^{n}$ viewed as a linear map has an eigenvalue of modulus 1 then it has a non-trivial core.

We should also note that while in this paper we are dealing with torsion free nilpotent groups by viewing them as lattices in their Mal'cev completions, Michael Kapovich in [9] has considered the case of a lattice in linear semisimple algebraic Lie group and proved that it admits a faithful self-similar action if and only if it is virtually isomorphic to an arithmetic lattice. It is an interesting open question when such a self-similar action is finite-state (see [9, Question 17]).

\section{Preliminary information about nilpotent Lie groups}

In this section we remind some facts from the theory of nilpotent Lie groups which we will need for the proof. Let $L$ be a connected and simply connected real nilpotent Lie group and $\mathcal{L}$ be its Lie algebra. The exponential map exp : $\mathcal{L} \rightarrow L$ is a diffeomorphism and one can define $\log : L \rightarrow \mathcal{L}$ as the inverse of exp.

Uniform subgroups. We say that $\mathcal{L}$ has a rational structure if there is a rational Lie subalgebra $\mathcal{L}_{\mathbb{Q}}$ such that $\mathcal{L}=\mathcal{L}_{\mathbb{Q}} \otimes \mathbb{R}$. A subgroup $G$ of $L$ is called uniform if it is discrete and $L / G$ is compact. The next theorem connects these two notions.

Theorem 4. (Theorem 5.1.8 (a) in [6]) Let L be a nilpotent Lie group with Lie algebra $\mathcal{L}$. If $L$ has a uniform subgroup $G$, then $\mathcal{L}$ has a rational structure such that $\mathcal{L}_{\mathbb{Q}}=\mathbb{Q}$-span $(\log G)$.

This theorem allows us, given a uniform subgroup $G$ of $L$, to fix the rational structure on $\mathcal{L}$ by defining $\mathcal{L}_{\mathbb{Q}}=\mathbb{Q}$-span $(\log G)$. The next theorem tells when two different uniforms subgroups define the same rational structure.

Theorem 5. (Theorem 5.1.12 in [6]) Let $G_{1}, G_{2}$ be uniform subgroups in a nilpotent Lie group $L$. Then $G_{1}, G_{2}$ determine the same rational structure in $\mathcal{L}$ if and only if they are commensurable: $G_{1} \cap G_{2}$ of finite index in both $G_{1}$ and $G_{2}$.

We will often consider subgroups of $G$ of finite index in $G$. It is clear they are still uniform subgroups of $L$, hence the above theorem tells that such a subgroup defines the same rational structure as $G$.

A uniform subgroup $G$ of $L$ is called a lattice subgroup if $\log G$ is an additive subgroup of $\mathcal{L}$. 
Theorem 6. (Theorem 5.4.2 (a) in [6]) Let $G$ be a uniform subgroup of a nilpotent Lie group $L$, then $G$ contains a lattice subgroup $G_{0}$ of finite index.

Mal'cev completion. Given a finitely generated torsion-free nilpotent group $G$, Mal'cev completion embeds $G$ as a uniform subgroup into connected and simply connected real nilpotent Lie group. This completion can be briefly described as follows. Since $G$ is nilpotent and torsion free, it is possible to add subgroups to its upper central series so that the obtained series, say $\{e\}=G_{1} \leq \cdots \leq G_{m+1}=G$, has infinite cyclic factors. By fixing a choice of $a_{i} \in G$ such that $a_{i} G_{i}$ generates $G_{i+1} / G_{i}$ for $i=1, \ldots, m$ we can write any $g \in G$ in the form $g=a_{m}^{t_{m}} \ldots a_{1}^{t_{1}}$ for some uniquely defined $t_{i} \in \mathbb{Z}$, called Mal'cev coordinates of $g$. Let $f: \mathbb{Z}^{m} \times \mathbb{Z}^{m} \rightarrow \mathbb{Z}^{m}$ and $g: \mathbb{Z}^{m} \rightarrow \mathbb{Z}^{m}$ be the maps that correspond to the product and taking inverse in $G$ in terms of coordinates. It can be shown that $f, g$ are polynomial, and hence have a natural extension from $\mathbb{Z}^{m}$ to $\mathbb{R}^{m}$. Moreover, they still satisfy the same laws, and hence define a nilpotent Lie group structure on $\mathbb{R}^{m}$ such that the subset $\mathbb{Z}^{m} \subset \mathbb{R}^{m}$ with induced group operations is a subgroup isomorphic to $G$.

Left invariant metrics on Lie groups. We recall the following construction of the left invariant Riemannian metrics on a Lie group $L$. Let $T_{g} L$ be the tangent space to $L$ at the point $g$, and recall that $\mathcal{L}=T_{e} L$. Denote by $l_{g}: T_{e} L \rightarrow T_{g} L$ the differential at the point $e$ of the map $x \mapsto g x$. Then any euclidean norm on $\mathcal{L}=T_{e} L$ gives rise to a left invariant Riemannian metric on $L$ by the rule $\|w\|=\left\|l_{g}^{-1} w\right\|$ for any $g \in L$ and $w \in T_{g} L$. Then the length of a differentiable curve $f(t) \in L, t \in[0,1]$ is defined as $\int_{0}^{1}\left\|f^{\prime}(t)\right\| d t$, and the distance $d\left(g, g^{\prime}\right)$ between $g, g^{\prime} \in L$ is the infimum of lengths of all curves that connect $g$ to $g^{\prime}$. Note that the length of the curve $f(t)$ is the same as the length of the shifted curve $g_{0} f(t)$ for any $g_{0} \in L$, and hence $d\left(g_{0} g, g_{0} g^{\prime}\right)=d\left(g, g^{\prime}\right)$.

\section{Proof of Theorems 2 and 3}

Recall that we have a finitely generated torsion-free nilpotent group $G$ and an isomorphism $\phi: H \rightarrow G$ from a subgroup $H<G$ of finite index with trivial $\phi$-core. The Mal'cev completion $L$ of $G$ is a nilpotent Lie group, connected and simply connected, and $G$ is a uniform subgroup of it. Thus all the theorems from the previous section apply. Note also that since $H$ is a subgroup of finite index in $G$, it is also a uniform subgroup of $L$. Therefore $L$ is also Mal'cev completion of $H$, by the uniqueness property of Mal'cev completion. The isomorphism $\phi: H \rightarrow G$ lifts to a diffeomorphic automorphism of $L$ and its differential $\widehat{\phi}$ at $e$ is an automorphism of Lie algebra $\mathcal{L}$. Under these notations we have

$$
\widehat{\phi}(\log (g))=\log (\phi(g)) \text { for all } g \in L .
$$

Before proving the theorems, let us show first that if $\widehat{\phi}$ is contracting, i.e., its spectral radius is less than one, then the associated self-similar actions of $G$ (independently on the choice of coset representatives) has a special contracting property. Namely, a self-similar action $\left(G, X^{*}\right)$ is called contracting if there exists a finite set $\mathcal{N} \subset G$ with the property 
that for every $g \in G$ there exists $n \in \mathbb{N}$ such that $\left.g\right|_{v} \in \mathcal{N}$ for all words $v \in X^{*}$ of length $\geq n$. Contracting property of a self-similar action doesn't depend on the choice of coset representatives and can be characterized just in terms of the associated virtual endomorphism (see [11, Proposition 2.11.11]).

Proposition 7. If $\widehat{\phi}$ is contracting, then every self-similar action of $(G, \phi)$ is contracting.

Proof. Let $\left(G, X^{*}\right)$ be a self-similar action associated to the triple $(G, \phi, D)$. Take any element $g \in G$ and for every word $x_{1} x_{2} \ldots x_{n} \in X^{*}$ consider the state

$$
\begin{aligned}
\left.g\right|_{x_{1} x_{2} \ldots x_{n}} & =\phi\left(q_{y_{n}}^{-1} \ldots \phi\left(q_{y_{2}}^{-1} \phi\left(q_{y_{1}}^{-1} g q_{x_{1}}\right) q_{x_{2}}\right) \ldots q_{x_{n}}\right) \\
& =\phi\left(q_{y_{n}}^{-1}\right) \ldots \phi^{n-1}\left(q_{y_{2}}^{-1}\right) \phi^{n}\left(q_{y_{1}}^{-1}\right) \phi^{n}(g) \phi^{n}\left(q_{x_{1}}\right) \phi^{n-1}\left(q_{x_{2}}\right) \ldots \phi\left(q_{x_{n}}\right),
\end{aligned}
$$

where $y_{1} y_{2} \ldots y_{n}=g\left(x_{1} x_{2} \ldots x_{n}\right)$. We use the following general lemma:

Lemma 1. Let $\lambda>0$ be bigger than the spectral radius of $\widehat{\phi}: \mathcal{L} \rightarrow \mathcal{L}$. Then the topology on $L$ is induced by a left invariant metric $d: L \times L \rightarrow \mathbb{R}_{+}$such that $d\left(\phi(g), \phi\left(g^{\prime}\right)\right) \leq \lambda d\left(g, g^{\prime}\right)$ for all $g, g^{\prime} \in L$.

Proof. It is easy to see that there is an euclidian norm on $\mathcal{L}$ such that $\|\widehat{\phi}(v)\| \leq \lambda\|v\|$ for any $v \in \mathcal{L}$. Then there is a unique left invariant Riemannian metric on $L$, induced by this norm. Moreover, by left invariance, for any vector $w$ tangent to $L$ we have that $\|\widehat{\phi}(w)\| \leq \lambda\|w\|$. Integrating, we obtain that $d\left(\phi(g), \phi\left(g^{\prime}\right)\right) \leq \lambda d\left(g, g^{\prime}\right)$ for all $g, g^{\prime} \in L$.

By the lemma there is $\lambda<1$ and a left-invariant metric $d$ on $L$ such that

$$
\begin{aligned}
& d\left(e, \phi^{n}\left(q_{x_{1}}\right) \phi^{n-1}\left(q_{x_{2}}\right) \ldots \phi\left(q_{x_{n}}\right)\right) \leq \\
\leq & d\left(e, \phi^{n}\left(q_{x_{1}}\right)\right)+d\left(\phi^{n}\left(q_{x_{1}}\right), \phi^{n}\left(q_{x_{1}}\right) \phi^{n-1}\left(q_{x_{2}}\right)\right)+\ldots \\
& \ldots+d\left(\phi^{n}\left(q_{x_{1}}\right) \phi^{n-1}\left(q_{x_{2}}\right) \ldots \phi^{2}\left(q_{x_{n-1}}\right), \phi^{n}\left(q_{x_{1}}\right) \phi^{n-1}\left(q_{x_{2}}\right) \ldots \phi\left(q_{x_{n}}\right)\right) \leq \\
\leq & \lambda^{n} d\left(e, q_{x_{1}}\right)+\cdots+\lambda d\left(e, q_{x_{n}}\right) \leq \frac{\lambda}{1-\lambda} \max _{i} d\left(e, q_{x_{i}}\right)=: R .
\end{aligned}
$$

Hence the set of all products of the form $\phi^{n}\left(q_{x_{1}}\right) \phi^{n-1}\left(q_{x_{2}}\right) \ldots \phi\left(q_{x_{n}}\right)$ belongs to a ball of finite radius $R$ in $L$, which is a compact set. Define the finite subset $\mathcal{N} \subset G$ as the set of all group elements in the ball of radius $2 R+1$ around identity in $L$. Since $\widehat{\phi}$ is contracting, $d\left(e, \phi^{n}(g)\right) \rightarrow 0$ as $n \rightarrow \infty$ and the product in (3) belongs to the ball of radius $2 R+1$ for all large enough $n \in \mathbb{N}$. Hence $\left.g\right|_{x_{1} x_{2} \ldots x_{n}} \in \mathcal{N}$ and the action is contracting.

It is obvious from definition that every contracting action is finite-state. Therefore Theorem 3 can be restated as follows: under the conditions of the theorem, all self-similar actions for $(G, \phi)$ are finite-state if and only if at least one of them (and hence every) is contracting.

Proof of sufficiency in Theorem 2. The assumption on the Jordan normal form of $\phi$ implies the following crucial property: for every $g \in L$ the sequence $\phi^{n}(g)$ is bounded (i.e., belongs to a compact set). 
It can be shown, using induction on the length of lower central series of $L$ that $[G$ : $H]=\operatorname{det} \widehat{\phi}^{-1}$.

The Lie algebra $\mathcal{L}$ decomposes in the direct sum $\mathcal{L}=\mathcal{L}_{c} \oplus \mathcal{L}_{r}$, where $\mathcal{L}_{c}$ is a $\phi$-invariant subalgebra such that $\left.\phi\right|_{\mathcal{L}_{c}}$ has spectral radius less than 1 (contracting), and the spectrum of $\left.\phi\right|_{\mathcal{L} / \mathcal{L}_{c}}$ consists only of numbers of modulus 1 . Consider the $\phi$-invariant subgroup $L_{c}=$ $\exp \left(\mathcal{L}_{c}\right)$ of the Lie group $L$ that corresponds to the subalgebra $\mathcal{L}_{c}$. One can define $L_{c}$ directly as the subgroup of all $g \in L$ such that $\phi^{n}(g) \rightarrow e$ as $n \rightarrow \infty$. Define the group $G_{c}=G \cap L_{c}$ and its subgroup $H_{c}=H \cap L_{c}=\phi^{-1}\left(G_{c}\right)<G_{c}$ of finite index $\left[G_{c}: H_{c}\right]=\operatorname{det}\left(\left.\phi\right|_{\mathcal{L}_{c}}\right)^{-1}$.

Notice that $\operatorname{det}\left(\left.\phi\right|_{\mathcal{L} / \mathcal{L}_{c}}\right)$ is positive as since $\operatorname{det}(\phi)=\operatorname{det}\left(\left.\phi\right|_{\mathcal{L} / \mathcal{L}_{c}}\right) \operatorname{det}\left(\left.\phi\right|_{\mathcal{L}_{c}}\right)$, and, at the same time, it is a product of numbers of modulus 1 . Hence $\operatorname{det}\left(\left.\phi\right|_{\mathcal{L} / \mathcal{L}_{c}}\right)=1$ and we get $[G: H]=\operatorname{det}(\phi)^{-1}=\operatorname{det}\left(\left.\phi\right|_{\mathcal{L}_{c}}\right)^{-1}=\left[G_{c}: H_{c}\right]$.

Take any coset representatives $q_{1}, q_{2}, \ldots, q_{d}$ for $H_{c}$ in $G_{c}$. Since $H \cap G_{c}=H_{c}$ and $[G: H]=\left[G_{c}: H_{c}\right]$, the elements $q_{1}, q_{2}, \ldots, q_{d}$ are also coset representatives of $H$ in $G$. Let us consider the associated self-similar action $\left(G, X^{*}\right)$ given by Equation (11). Take any element $g \in G$ and for every word $x_{1} x_{2} \ldots x_{n} \in X^{*}$ consider the state

$$
\begin{aligned}
\left.g\right|_{x_{1} x_{2} \ldots x_{n}} & =\phi\left(q_{y_{n}}^{-1} \ldots \phi\left(q_{y_{2}}^{-1} \phi\left(q_{y_{1}}^{-1} g q_{x_{1}}\right) q_{x_{2}}\right) \ldots q_{x_{n}}\right) \\
& =\phi\left(q_{y_{n}}^{-1}\right) \ldots \phi^{n-1}\left(q_{y_{2}}^{-1}\right) \phi^{n}\left(q_{y_{1}}^{-1}\right) \phi^{n}(g) \phi^{n}\left(q_{x_{1}}\right) \phi^{n-1}\left(q_{x_{2}}\right) \ldots \phi\left(q_{x_{n}}\right),
\end{aligned}
$$

where $y_{1} y_{2} \ldots y_{n}=g\left(x_{1} x_{2} \ldots x_{n}\right)$. The sequence $\phi^{n}(g)$ is bounded in $L$. The elements $q_{x_{1}}, q_{x_{2}}, \ldots, q_{x_{n}}$ are taken from a finite subset of the $\phi$-invariant subgroup $L_{c}$. Then the set of all products of the form $\phi^{n}\left(q_{x_{1}}\right) \phi^{n-1}\left(q_{x_{2}}\right) \ldots \phi\left(q_{x_{n}}\right)$ belongs to a compact subset of $L_{c}$ (see the proof of Proposition 7). Since the product in (44) belongs to the lattice $G$, it assumes a finite number of values. Hence the action $\left(G, X^{*}\right)$ is finite-state.

Proof of necessity in Theorem Q Let $\left(G, X^{*}\right)$ be a finite-state self-similar action with virtual endomorphism $\phi$ associated to the letter $x_{1} \in X$, i.e., $\phi=\phi_{x_{1}}$ and $H=S t_{G}\left(x_{1}\right)$. Let $\left\{q_{1}=e, q_{2}, \ldots, q_{d}\right\}$ be the corresponding set of coset representatives.

Lemma 2. The eigenvalues of $\widehat{\phi}$ have modulus $\leq 1$. Moreover, every eigenvalue of modulus 1 is a root of unity.

Proof. Put $\mathcal{L}^{(0)}=\mathcal{L}$ and let $\mathcal{L}^{(i)}=\left[\mathcal{L}, \mathcal{L}^{(i-1)}\right]$ be the lower central series of the Lie algebra $\mathcal{L}$. Since $\widehat{\phi}$ is an automorphism of $\mathcal{L}$ it preserves every term $\mathcal{L}^{(i)}$ and induces an automorphism $\varphi_{i}$ on the quotient $\mathcal{L}^{(i)} / \mathcal{L}^{(i+1)}$. The spectrum of $\widehat{\phi}$ is a union of the spectra of $\varphi_{i}$. At the same time, every linear map $\varphi_{i}$ is a quotient of the tensor product $\varphi_{0} \otimes \varphi_{0} \otimes \ldots \otimes \varphi_{0}$ (see [14, Theorem 3.1]). Hence it is enough to prove the statement for the automorphism $\varphi_{0}$.

Let $\lambda$ be an eigenvalue of $\varphi_{0}$. Take a basis of $\mathcal{L} /[\mathcal{L}, \mathcal{L}]$ in which $\varphi_{0}$ has Jordan normal form, and consider the coordinate of vectors in this basis that corresponds to an eigenvector with eigenvalue $\lambda$. There exists a linear map $\xi: \mathcal{L} \rightarrow \mathbb{C}$ such that $\xi([\mathcal{L}, \mathcal{L}])=0$ and $\xi(\phi(l))=\lambda \xi(l)$ for all $l \in \mathcal{L}$. We compose $\xi$ with the logarithmic map $\log : L \rightarrow \mathcal{L}$ and denote the composition also by $\xi$. Note that $\log \left(g_{1} g_{2}\right)=\log \left(g_{1}\right)+\log \left(g_{2}\right) \bmod [\mathcal{L}, \mathcal{L}]$. Thus we have a map $\xi: L \rightarrow \mathbb{C}$ such that $\xi\left(g_{1} g_{2}\right)=\xi\left(g_{1}\right)+\xi\left(g_{2}\right)$ and $\xi(\phi(g))=\lambda \xi(g)$. The rest of the proof is very similar to the proof of Theorem 2.12.1 in [11], so we only sketch it here. 
Since $G$ is a lattice in $L$, there exists $g \in G$ such that $\xi(g) \neq 0$. Let us consider the states $\left.g\right|_{v}$ for $v \in X^{*}$. By Equation (1) we have $\left.g\right|_{x}=\phi\left(q_{g(x)}^{-1} g q_{x}\right)$ for every $x \in X$. Then

$$
\xi\left(\left.g\right|_{x}\right)=\lambda \xi(g)+\lambda\left(\xi\left(q_{x}\right)-\xi\left(q_{g(x)}\right)\right)=\lambda \xi(g)+d_{x}
$$

where $d_{x}=\lambda\left(\xi\left(q_{x}\right)-\xi\left(q_{g(x)}\right)\right)$.

Suppose $|\lambda|>1$. Since $\sum_{x \in X} d_{x}=0$, it follows that there exits $x_{1} \in X$ such that $\left|\xi\left(\left.g\right|_{x_{1}}\right)\right|>|\xi(g)|$. Thus we can iteratively construct letters $x_{n} \in X$ such that $\left|\xi\left(\left.g\right|_{x_{1} x_{2} \ldots x_{n+1}}\right)\right|>\left|\xi\left(\left.g\right|_{x_{1} x_{2} \ldots x_{n}}\right)\right|$ for each $n$. Hence $g$ is not finite-state, contradiction.

Suppose $|\lambda|=1$ and $\lambda$ is not a root of unity. As above there is a sequence of letters $x_{n} \in X$ such that for each $n$ either $\left|\xi\left(\left.g\right|_{x_{1} x_{2} \ldots x_{n+1}}\right)\right|>\left|\xi\left(\left.g\right|_{x_{1} x_{2} \ldots x_{n}}\right)\right|$ or $\xi\left(\left.g\right|_{x_{1} x_{2} \ldots x_{n+1}}\right)=$ $\lambda \xi\left(\left.g\right|_{x_{1} x_{2} \ldots x_{n}}\right)$. In either case we have a contradiction with the fact that the action is finitestate.

It is left to prove that Jordan cells for roots of unity have size 1 . Let $m$ be an integer number such that $\varepsilon^{m}=1$ for every root of unity $\varepsilon$ from the spectrum of $\widehat{\phi}$. Then the spectrum of $\widehat{\phi}^{m}$ consists of 1 and numbers less than 1 in modulus. The self-similar action $\left(G, X^{*}\right)$ over the alphabet $X$ induces the self-similar action $\left(G,\left(X^{m}\right)^{*}\right)$ over the alphabet $X^{m}$ of words of length $m$ over $X$. Moreover, since the action $\left(G, X^{*}\right)$ is finite-state then obviously the action $\left(G,\left(X^{m}\right)^{*}\right)$ is also finite-state. Note that the virtual endomorphism $\phi_{v}$ of the action $\left(G,\left(X^{m}\right)^{*}\right)$ associated to a word $v=x_{1} \ldots x_{m} \in X^{m}$ is the composition $\phi_{v}=\phi_{x_{1}} \circ \cdots \circ \phi_{x_{n}}$ of virtual endomorphisms of the action over $X$. In particular, $\phi_{x_{1} \ldots x_{1}}=\phi^{m}$ and the action $\left(G,\left(X^{m}\right)^{*}\right)$ corresponds to the pair $\left(G, \phi^{m}\right)$. If we know that the size of Jordan cells of $\widehat{\phi}^{m}$ with eigenvalue 1 have size 1 , then the same holds for $\widehat{\phi}$ for roots of unity. Hence we can assume that all roots of unity in the spectrum of $\widehat{\phi}$ are equal to 1 .

Recall that $\mathcal{L}_{\mathbb{Q}}=\mathbb{Q}$-span $(\log G)$ is a rational Lie subalgebra of $\mathcal{L}$ by Theorem 4 such that $\mathcal{L}_{\mathbb{Q}} \otimes \mathbb{R}=\mathcal{L}$ (i.e., determines a rational structure on $\mathcal{L}$ ). It is easy to see that $\widehat{\phi}\left(\mathcal{L}_{\mathbb{Q}}\right) \subset \mathcal{L}_{\mathbb{Q}}$. Indeed, since $H$ is of finite index in $G$ they are commensurable and hence by Theorem 5 define the same rational structure on $\mathcal{L}$. Thus by Theorem $4 \mathcal{L}_{\mathbb{Q}}$ is also equal to the $\mathbb{Q}$-span of vectors from $\log (H)$. Since $\phi(H) \subset G$ we have that $\widehat{\phi}\left(\mathcal{L}_{\mathbb{Q}}\right) \subset \mathcal{L}_{\mathbb{Q}}$ by Equation (2).

Suppose there is a Jordan cell of $\widehat{\phi}$ with eigenvalue 1 that has size greater than 1 . Then there exist nonzero vectors $v, u \in \mathcal{L}$ such that $\widehat{\phi}(v)=v$ and $\widehat{\phi}(u)=u+v$. Since $\widehat{\phi}\left(\mathcal{L}_{\mathbb{Q}}\right) \subset \mathcal{L}_{\mathbb{Q}}$, the vectors $v$ and $u$ can be chosen in $\mathcal{L}_{\mathbb{Q}}$. By Theorem 6 the group $G$ contains a subgroup $G_{0}$ of finite index such that $\log G_{0}$ is a lattice in $\mathcal{L}_{\mathbb{Q}}$, i.e., $\log G_{0}$ is closed under addition and its span over $\mathbb{Q}$ is equal to $\mathcal{L}_{\mathbb{Q}}$. Multiplying $v$ and $u$ by a suitable integer we can assume that they belong to $\log G_{0}$, and thus $u+n v \in \log G_{0}$ for all $n \in \mathbb{N}$. Consider the element $g=\exp (u) \in G$. We get

$$
\phi^{n}(g)=\phi^{n}(\exp (u))=\exp \left(\widehat{\phi}^{n}(u)\right)=\exp (u+n v) \in G_{0} \subset G,
$$

and hence $\phi^{n-1}(g) \in \phi^{-1}(G)=H=S t_{G}\left(x_{1}\right)$ for all $n \geq 1$. Then the element $g$ fixes the word $x_{1} x_{1} \ldots x_{1}$ ( $n$ times) and has the state $\left.g\right|_{x_{1} x_{1} \ldots x_{1}}=\phi^{n}(g)=\exp (u+n v)$ for all $n \geq 1$. Since all elements $u+n v$ are different, the element $g$ is not finite-state. We got a contradiction. 
Proof of Theorem 3. If the spectral radius of $\widehat{\phi}$ is less than 1 , then the action is contracting by Proposition 7 and thus finite-state.

For the converse, it is sufficient to prove that if the Jordan normal form of $\widehat{\phi}$ satisfies the condition in Theorem 2 and the spectrum of $\widehat{\phi}$ contains a root of unity then there exists a non-finite-state action for $(G, \phi)$. As in the previous proof, we can assume that all roots of unity from the spectrum are equal to 1 , and we find an element $q \in H$ such that $\phi(q)=q$. Since the $\phi$-core is trivial, the $\left.\phi\right|_{Z(H)^{-c o r e}}$ is trivial too by Proposition 1, Then by [11, Theorem 2.12.1] the corresponding linear map $\left.\widehat{\phi}\right|_{Z(H)}$ has spectral radius less than 1. Let us choose a set of coset representatives $D=\left\{q_{1}=e, q_{2}, \ldots, q_{d^{\prime}}\right\}$ for $Z(H)$ in $Z(G)$. If every element $g \in Z(G)$ can be expressed as a product

$$
\begin{aligned}
g & =q_{i_{1}} \phi^{-1}\left(q_{i_{2}} \phi^{-1}\left(\ldots \phi^{-1}\left(q_{i_{n}}\right) \ldots\right)\right)= \\
& =q_{i_{1}} \phi^{-1}\left(q_{i_{2}}\right) \phi^{-2}\left(q_{i_{3}}\right) \ldots \phi^{-n+1}\left(q_{i_{n}}\right)
\end{aligned}
$$

for $q_{i_{j}} \in D$, then we take $k>1$ such that the set $D^{k}=\left\{q_{1}^{k}=e, q_{2}^{k}, \ldots q_{d^{\prime}}^{k}\right\}$ consists of coset representatives for $Z(H)$ in $Z(G)$ and replace $D$ by $D^{k}$. In this case every product in (5) belongs to a proper subgroup $Z(G)^{k}$ of $Z(G)$. We complete $D$ to the set of coset representatives of $H$ in $G$ by elements $q_{d^{\prime}+1}, \ldots, q_{d}$. Replace the coset representative $q_{1}=e$ by the element $q \in H$. Let us prove that the associated self-similar action of the group $G$ is not finite-state.

Take an element $g \in Z(G)$ that cannot be expressed in the form (5), and consider the state of $g$ at the word $x_{1} x_{1} \ldots x_{1}$ ( $n$ times):

$$
\left.g\right|_{x_{1} x_{1} \ldots x_{1}}=\phi\left(q_{y_{n}}^{-1} \ldots \phi\left(q_{y_{2}}^{-1} \phi\left(q_{y_{1}}^{-1} g q\right) q\right) \ldots q\right)=\phi\left(q_{y_{n}}^{-1} \ldots \phi\left(q_{y_{2}}^{-1} \phi\left(q_{y_{1}}^{-1} g\right)\right) \ldots\right) q^{n}
$$

where $g\left(x_{1} x_{1} \ldots x_{1}\right)=y_{1} y_{2} \ldots y_{n}$. All elements $q_{y_{i}}$ are taken from the set $\left\{q, q_{2}, \ldots, q_{d^{\prime}}\right\}$. The elements $\phi\left(q_{y_{j}}^{-1} \ldots \phi\left(q_{y_{2}}^{-1} \phi\left(q_{y_{1}}^{-1} g\right)\right) \ldots\right)$ with all $q_{y_{k}}$ from $\left\{q_{2}, \ldots, q_{d^{\prime}}\right\}$ belong to the center $Z(G)$. We can move every element $q_{y_{i}}$ that is equal to $q$ to the right and include in the power $q^{n}$. Hence we can write the state as

$$
\left.g\right|_{x_{1} x_{1} \ldots x_{1}}=\phi\left(q_{y_{n}}^{-1} \ldots \phi\left(q_{y_{2}}^{-1} \phi\left(q_{y_{1}}^{-1} g\right)\right) \ldots\right) q^{m}
$$

with all $q_{y_{i}} \in\left\{e, q_{2}, \ldots, q_{d^{\prime}}\right\}$ (here we replace every $q_{y_{i}}=q$ by $q_{y_{i}}=e$ ), and $m$ is equal to the number of letters $y_{i}$ not equal to $x_{1}$. Since $\left.\widehat{\phi}\right|_{Z(H)}$ is contracting, all the products $\phi\left(q_{y_{n}}^{-1}\right) \ldots \phi^{n-1}\left(q_{y_{2}}^{-1}\right) \phi^{n}\left(q_{y_{1}}^{-1}\right) \phi^{n}(g)$ belong to a compact subset of $L$ (see the proof of Proposition 7), but also belong to the lattice $G$. Hence these products assume a finite number of values. Let us analyze the values of $m$.

Notice that $g$ cannot stabilize the sequence $x_{1} x_{1} \ldots$ Indeed, if $g\left(x_{1}\right)=x_{1}$ then $\left.g\right|_{x_{1}}=$ $\phi\left(q^{-1} g q\right)=\phi(g) \in Z(G)$. Hence, if $g\left(x_{1} x_{1} \ldots\right)=x_{1} x_{1} \ldots$ then $\phi^{n}(g) \in Z(G)$ for all $n \geq 1$. It implies that there exists a non-trivial normal $\phi$-invariant subgroup of $Z(G)$ and we get a contradiction with the faithfulness of the action. Suppose $g$ changes only finitely many letters in the sequence $x_{1} x_{1} \ldots$ Then $\left.g\right|_{x_{1} x_{1} \ldots x_{1}}$ and $\left.g\right|_{x_{1} x_{1} \ldots x_{1}} q^{-m}$ stabilize $x_{1} x_{1} \ldots$ for long enough word $x_{1} x_{1} \ldots x_{1}$. At the same time

$$
\left.g\right|_{x_{1} x_{1} \ldots x_{1}} q^{-m}=\phi\left(q_{y_{n}}^{-1} \ldots \phi\left(q_{y_{2}}^{-1} \phi\left(q_{y_{1}}^{-1} g\right)\right) \ldots\right) \in Z(G) .
$$


and we get that this element should be trivial. However in this case $g$ can be expressed in the form (5), we get a contradiction with the choice of $g$.

Since $g$ changes infinitely many letters in the sequence $x_{1} x_{1} \ldots$, the number $m$ in Equation (6) goes to infinity as the length of $x_{1} x_{1} \ldots x_{1}$ goes to infinity. The elements $q^{m}$ are all different, and hence $g$ has infinitely many states.

\section{Example}

Consider the discrete Heisenberg group

$$
G=\left\{(x, y, z)=\left(\begin{array}{ccc}
1 & x & z \\
0 & 1 & y \\
0 & 0 & 1
\end{array}\right): x, y, z \in \mathbb{Z}\right\}
$$

its subgroup $H=\{(x, 2 y, 2 z): x, y, z \in \mathbb{Z}\}$, and the isomorphism $\phi: H \rightarrow G$ given by $\phi(x, y, z)=(x, y / 2, z / 2)$. One can directly check that the $\phi$-core is trivial, and therefore every self-similar action for the pair $(G, \phi)$ is faithful (we can also notice that $\left.\phi\right|_{Z(H)}$ : $Z(H) \rightarrow Z(G)$ has spectral radius $\frac{1}{2}$ and thus self-similar actions for $\left(Z(G),\left.\phi\right|_{Z(H)}\right)$ are faithful, and hence the same holds for $(G, \phi)$ by Proposition 11).

The matrix of $\phi$ is diagonal with eigenvalues $1, \frac{1}{2}, \frac{1}{2}$, and Theorems 2 and 3 imply that there exist both finite-state and non-finite-state self-similar actions for the pair $(G, \phi)$. First, let us construct a finite-state action. Choose coset representatives $D=\{(0,0,0),(0,1,0),(0,0,1),(0,1,1)\}$ and consider the associated self-similar action $\left(G, X^{*}\right)$ over the alphabet $X=\{1,2,3,4\}$ given by Equation (1). The action of the generators $a=(1,0,0)$ and $b=(0,1,0)$ of the group satisfies the following recursions:

$$
\begin{array}{llll}
a(1 v)=1 a(v) & a(2 v)=4 a(v) & a(3 v)=3 a(v) & a(4 v)=2\left(b^{-1} a b\right)(v) \\
b(1 v)=2 v & b(2 v)=1 b(v) & b(3 v)=4 v & b(4 v)=3 b(v)
\end{array}
$$

The elements $a$ and $b$ are finite-state, namely the states of $a$ are $a, b^{-1} a b, b^{-2} a b^{2}$ and the states of $b$ are $e, b$. Hence the action $\left(G, X^{*}\right)$ is finite-state.

Let us change the set of coset representatives and choose $D^{\prime}=\{(1,0,0)$, $(0,1,0),(0,0,1),(0,1,1)\}$. Then the action of the generators of the group $G$ satisfies the recursions

$$
\begin{aligned}
& a(1 v)=1 a(v) \quad a(2 v)=4 a(v) \quad a(3 v)=3 a(v) \quad a(4 v)=2\left(b^{-1} a b\right)(v) \\
& b(1 v)=2 a(v) \quad b(2 v)=1\left(a^{-1} b\right)(v) \quad b(3 v)=4 v \quad b(4 v)=3 b(v)
\end{aligned}
$$

In order to see that this action is not finite-state, let us look at the action of the element $c=(0,0,1)=a^{-1} b^{-1} a b$ :

$$
c(1 v)=3\left(a^{2} b^{-1} a^{-1} b\right)(v) \quad c(2 v)=4 c(v) \quad c(3 v)=1 a^{-1}(v) \quad c(4 v)=2 c^{2}(v)
$$

The element $c^{2}$ is a state of $c$, and $\left.c^{2 n}\right|_{2}=c^{3 n},\left.c^{2 n+1}\right|_{2}=c^{3 n+1},\left.c^{2 n+1}\right|_{4}=c^{3 n+2}$. Inductively we get that all powers $c^{n}$ for $n \in \mathbb{N}$ are the states of $c$ and hence the element $c$ is not finite-state. 


\section{References}

[1] Bartholdi, L., Grigorchuk, R.: On the spectrum of Hecke type operators related to some fractal groups. Proc. Steklov Inst. Math. 231, 1-41 (2000)

[2] Bartholdi, L., Sunik, Z.: Some solvable automaton groups. Contemporary Mathematics 394, 11-29 (2006)

[3] Berlatto, A., Sidki, S.: Virtual endomorphisms of nilpotent groups. Groups Geom. Dyn. 1(1), 21-46 (2007)

[4] Bondarenko, I.V.: Growth of Schreier graphs of automaton groups. Mathematische Annalen 354(2), 765-785 (2012)

[5] Brunner, A.M., Sidki, S.: The generation of $G L(n, \mathbb{Z})$ by finite state automata. Internat. J. Algebra Comput. 8(1), 127-139 (1998)

[6] Corwin, L.J., Greenleaf, F.P.: Representations of nilpotent Lie groups and their applications. Part 1: Basic theory and examples. Cambridge Studies in Advanced Mathematics, 18. Cambridge etc.: Cambridge University Press (1990)

[7] Grigorchuk, R.I., Nekrashevych, V.V., Sushchansky, V.I.: Automata, dynamical systems and groups. Proceedings of the Steklov Institute of Mathematics 231, 128-203 (2000)

[8] Grigorchuk, R.I., Żuk, A.: The lamplighter group as a group generated by a 2-state automaton, and its spectrum. Geom. Dedicata 87(1-3), 209-244 (2001)

[9] Kapovich, M.: Arithmetic aspects of self-similar groups. To appear in Groups, Geometry and Dynamics.

[10] Malcev, A.I.: On a class of homogeneous spaces. Izv. Akad. Nauk SSSR Ser. Mat. 13, 9-32 (1949)

[11] Nekrashevych, V.: Self-similar groups. Mathematical Surveys and Monographs, vol.117, American Mathematical Society, Providence (2005)

[12] Nekrashevych, V., Sidki, S.: Automorphisms of the binary tree: state-closed subgroups and dynamics of 1/2-endomorphisms. In T.W.Muller, editor, Groups: Topological, Combinatorial and Arithmetic Aspects, volume 311 of LMS Lecture Notes Series, 375-404 (2004)

[13] Savchuk, D., Vorobets, Y.: Automata generating free products of groups of order 2. Journal of Algebra 336(1), 53-66 (2011)

[14] Warfield, R.B.: Nilpotent groups. Lecture Notes in Mathematics 513, BerlinHeidelberg-New York: Springer-Verlag (1976) 